\title{
THE ACCESSIBLE SETS OF QUADRATIC FREE NILPOTENT CONTROL SYSTEMS*
}

\author{
ARTHUR J. KRENER ${ }^{\dagger}$
}

\begin{abstract}
We consider the problem of describing the accessible set of some simple control systems, the free nilpotent ones. We review what is known about linear free nilpotent control systems and the simplest quadratic free nilpotent control system. In these cases the accessible sets are cell complexes and the boundary cells are completely known. We are particularly interested in a quadratic free nilpotent system that is a extension of the famous system of Fuller. In this case the accessible sets are harder to describe because there are boundary points that can only be reached by chattering bang bang trajectories. A bang bang trajectory is chattering if it has an infinite number of switches in finite time. We offer some insights and conjectures about the accessible sets of the extended Fuller system.
\end{abstract}

Key Words: Free nilpotent control system, bang bang trajectory, chattering, singular arcs, Fuller's system.

1. Introduction. This paper is dedicated to my esteemed colleague and good friend John Baillieul. I first met John in 1974 when he was a graduate student and I was a post doc working with Roger Brockett at Harvard. This was in the early days of the geometric approach to nonlinear control and the accessibility problem was an important one because it was so fundamental and lent itself to differential geometric techniques.. It is also a very complicated one and remains largely open to this day. So in honor of John I have decided to take another crack at it and hopefully add something to our knowledge in this area.

One of the fundamental mathematical problems of control theory is to describe the accessible sets of a control system. For simplicity of discussion we shall restrict our attention to systems affine in the control

$$
\dot{x}=f(x)+g(x) u
$$

where $x \in \mathbb{R}^{n}, u \in \mathrm{U} \subset \mathbb{R}^{m}$.

Given an initial state $x^{0}$ we are interested in describing the set of points $\mathrm{A}\left(x^{0}\right)$ that are accessible from $x^{0}$ in forward time,

$$
\mathrm{A}\left(x^{0}\right)=\{x(t): t \geq 0, u(0: t) \text { bounded and measurable, } u(t) \in \mathrm{U}\}
$$

and the set of points $\mathrm{A}_{t}\left(x^{0}\right)$ accessible exactly at time $t>0$

$$
\mathrm{A}_{t}\left(x^{0}\right)=\{x(t): u(0: t) \text { bounded and measurable, } u(t) \in \mathrm{U}\}
$$

${ }^{*}$ Dedicated to John Baillieul on the Occasion of His 65th Birthday. Research supported in part by AFOSR.

$\dagger$ Department of Applied Mathematics, Naval Postgraduate School, Monterey, CA 93943-5216, USA. E-mail: ajkrener@nps.edu 
The notation $u(0: t)$ denotes the control trajectory $s \mapsto u(s)$ for $s \in[0, t]$.

The simplest example of this question is for a linear system of the form

$$
\dot{x}=F x+G u
$$

where $\mathrm{U}=\mathbb{R}^{m}$. Then it is well-known that

$$
\mathrm{A}_{t}\left(x^{0}\right)=e^{F t} x^{0}+\text { column span }\left[\begin{array}{llll}
G & F G & \ldots & F^{n-1} G
\end{array}\right]
$$

Hence if the above columns span $\mathbb{R}^{n}$ then $\mathrm{A}_{t}\left(x^{0}\right)=\mathbb{R}^{n}$.

Early on it was shown that if the smallest Lie algebra containing the vector fields $f$ and $g^{1}, \ldots, g^{n}$ spans the tangent space at every $x$ then the accessible set $\mathrm{A}\left(x^{0}\right)$ is between an open set and its closure [2], [8]. Sussman and Jurdjevic also showed that if the smallest ideal of this Lie algebra that contains the vector field $g$ spans the tangent space at every $x$ then the accessible set $\mathrm{A}_{\mathrm{t}}\left(x^{0}\right)$ at time $t>0$ is between an open set and its closure [8].

If the control is restricted to lie in a proper subset $U$ of $\mathbb{R}^{m}$ the geometry of the accessible sets is more interesting. Recently [7] we showed that the accessible sets for the systems that are strings of integrators

$$
\begin{aligned}
\dot{x}_{1} & =u \\
\dot{x}_{2} & =x_{1} \\
& \vdots \\
\dot{x}_{n} & =x_{n-1}
\end{aligned}
$$

with scalar control bounded in norm $|u| \leq 1$ are cell complexes. To describe these cell complexes we need some terminology.

Assume that $\mathrm{U}$ is a bounded convex subset of $\mathbb{R}^{m}$. A control trajectory $u\left(t_{1}: t_{2}\right)$ is bang bang if the control $u(t)$ at almost any time $t \in\left[t_{1}, t_{2}\right]$ is an extreme point of $\mathrm{U}$. For example if $\mathrm{U}=[-1,1]$ then a control trajectory is bang bang if $|u(t)|=1$ for almost all $t \in\left[t_{1}, t_{2}\right]$.

We denote by $\mathrm{A}_{t}^{n}\left(x^{0}\right)$ the accesible set at time $t>0$ for the string of $n$ integrators (1.2). We showed in [7] that every state $x \in \mathrm{A}_{t}^{n}\left(x^{0}\right)$ is reachable by using a bang bang control trajectory with no more than $n-1$ switches between \pm 1 . Every state in the interior of $\mathrm{A}_{t}^{n}\left(x^{0}\right)$ is reachable by a bang bang control trajectory of total time $t$ with exactly $n-1$ switches.

Consider the $k$ dimensional simplex

$$
\mathrm{S}_{t}^{k}=\left\{\left(s_{0}, \ldots, s_{k}\right) \mid s_{i} \geq 0, t=s_{0}+\cdots+s_{k}\right\}
$$

To each point in the interior of this simplex we can associate two bang bang control trajectories with exactly $k$ switches. One trajectory starts with $u(t)=1$ on $\left[0, s_{0}\right]$ and 
the other starts with $u(t)=-1$ on $\left[0, s_{0}\right]$. Let $\mathrm{R}_{ \pm}^{n}\left(s_{0}, \ldots, s_{k}\right)$ denote the endpoints at time $t$ of the corresponding state trajectories started at $x^{0}$ for the system (1.2).

The mappings

$$
\begin{aligned}
& \left(s_{0}, \ldots, s_{n}\right) \mapsto \mathrm{R}_{+}^{n}\left(s_{0}, \ldots, s_{n}\right) \\
& \left(s_{0}, \ldots, s_{n}\right) \mapsto \mathrm{R}_{-}^{n}\left(s_{0}, \ldots, s_{n}\right)
\end{aligned}
$$

are two diffeomorphisms from the interior of $\mathrm{S}_{t}^{n}$ onto the interior of $\mathrm{A}_{t}^{n}\left(x^{0}\right)$ so the interior of $\mathrm{A}_{t}^{n}\left(x^{0}\right)$ is a cell of dimension $n$.

The boundary of $\mathrm{A}_{t}^{n}\left(x^{0}\right)$ consists of two cells of dimension $n-1$ which intersect in two cells of dimension $n-2$ which intersect in two cells of dimension $n-3$, etc., which intersect in two cell of dimension 0 . The interiors of the two cells of dimension $n-1$ are the diffeomorphic images of the interior of $\mathrm{S}_{t}^{n-1}$ under the mappings

$$
\begin{aligned}
\left(s_{0}, \ldots, s_{n-1}\right) & \mapsto \mathrm{R}_{+}^{n}\left(s_{0}, \ldots, s_{n-1}\right) \\
\left(s_{0}, \ldots, s_{n-1}\right) & \mapsto \mathrm{R}_{-}^{n}\left(s_{0}, \ldots, s_{n-1}\right)
\end{aligned}
$$

The two cells of dimension $n-2$ are the diffeomorphic images of the interior of $\mathrm{S}_{t}^{n-2}$ under the mappings

$$
\begin{aligned}
\left(s_{0}, \ldots, s_{n-2}\right) & \mapsto \mathrm{R}_{+}^{n}\left(s_{0}, \ldots, s_{n-2}\right) \\
\left(s_{0}, \ldots, s_{n-2}\right) & \mapsto \mathrm{R}_{-}^{n}\left(s_{0}, \ldots, s_{n-2}\right)
\end{aligned}
$$

and so on. The two cells of dimension 0 are the points $\mathrm{R}_{ \pm}^{n}(t)$.

Figure 1 shows two views of $\mathrm{A}_{1}^{3}(0)$. The interior of $\mathrm{A}_{1}^{3}(0)$ is the image of the interior of $\mathrm{S}_{t}^{3}$ under either of the maps $\mathrm{R}_{ \pm}^{3}\left(s_{0}, s_{1}, s_{2}, s_{3}\right)$. The upper bounding 2-cell is the image of the interior of $\mathrm{S}_{t}^{2}$ under the map $\mathrm{R}_{+}^{3}\left(s_{0}, s_{1}, s_{2}\right)$ and the lower bounding 2-cell is the image of the interior of $\mathrm{S}_{t}^{2}$ under the map $\mathrm{R}_{-}^{3}\left(s_{0}, s_{1}, s_{2}\right)$. The two 2-cells intersect in two 1-cells which intersect in two 0-cells. Notice that the variation in the first coordinate is larger than the variation in the second coordinate which in turn is larger than the variation in the third coordinate.

The goal of this paper is to give similar descriptions of the accessible sets of nonlinear modifications of the string of integrators, systems of the form

$$
\begin{aligned}
\dot{x}_{1} & =u \\
\dot{x}_{2} & =x_{1} \\
& \vdots \\
\dot{x}_{n} & =x_{n-1} \\
\dot{x}_{n+1} & =\frac{x_{k}^{2}}{2}
\end{aligned}
$$

The accessible sets of such system are quite a bit more complicated and we are only able to fully describe them when $k=1, n=2$. This description was given by 

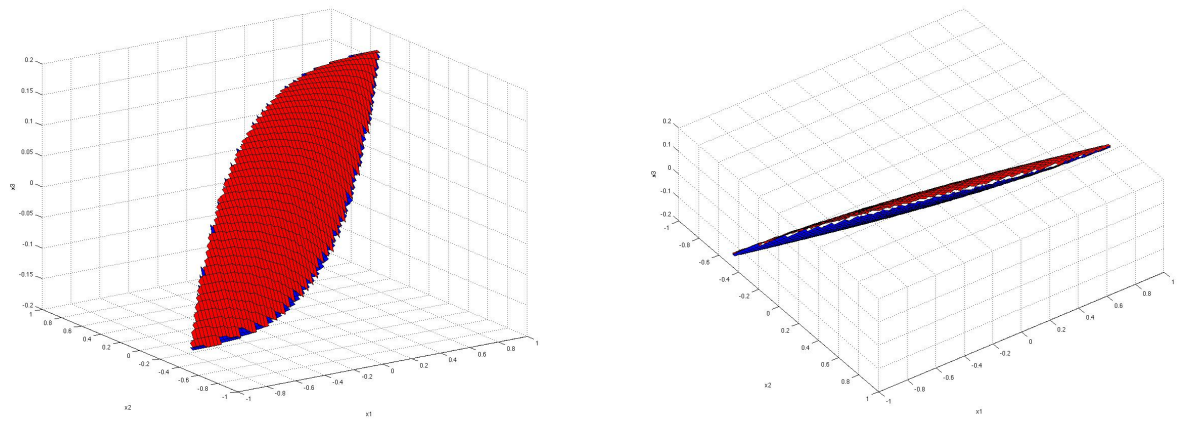

FIG. 1. $\mathrm{A}_{1}^{3}(0)$, top and side views.

Krener and Schaettler in [6]. When $k=2, n=2$ we have the famous system of A. T. Fuller [1]. The Fuller system is remarkable because some points of the accessible set are only reachable by chattering bang bang controls. A bang bang control trajectory is chattering if there are an infinite number of switches in a finite time interval. The accessible sets of the Fuller system and its extension $k=2, n=4$ are quite complicated. In this paper we shall offer some insights into their structures.

The rest of the paper is as follows. In the next section we introduce the concept of a free nilpotent system and explain why these are a nice class of systems for which to address the accessibility problem. The linear strings of integrators (1.2) and the nonlinear systems (1.3) with $n \geq 2 k$ are examples of free nilpotent systems. In Section 3 we consider linear free nilpotent systems like the string of integrators (1.2) and in Section 5 we consider quadratic free nilpotent systems like (1.3). The important tool for finding the boundary of an accessible set is the Pontryagin Maximum Principle which is discussed in Section 4. Section 6 reviews the results of [6] for the quadratic free nilpotent system (1.3) with $n=2$ and $k=1$. In Section 7 we offer some insights into the structure of the accessible sets or the quadratic free nilpotent system (1.3) with $n=4$ and $k=2$. We close with some conclusions.

2. Free Nilpotent Control Systems. We expect that for some simple control systems the accessible sets are nice cell complexes. But what do we mean by simple control systems. If we were to ask what are simple functions, an obvious answer are the polynomials. And why are they simple, one possible answer is that all their higher derivatives are zero. With this as motivation we might call a control system simple if all its higher derivatives are zero. But what do we mean by the derivatives of a control system? The concept should be coordinate free so we take derivatives to be the Lie bracket of the vector fields $f$ and $g$. We define the Lie bracket of two vector fields to be

$$
[f, g](x)=\frac{\partial f}{\partial x}(x) g(x)-\frac{\partial g}{\partial x}(x) f(x)
$$


This is the negative of the standard definition but this one (2.1) simplifies some formulas by eliminating minus signs. The Lie bracket is skew-symmetric

$$
[f, g]=-[g, f]
$$

and satisfies the Jacobi identity

$$
[f,[g, h]]+[g,[h, f]]+[h,[f, g]]=0
$$

The three Lie jet at $x^{0}$ of the control system (1.1) is the tree of vectors

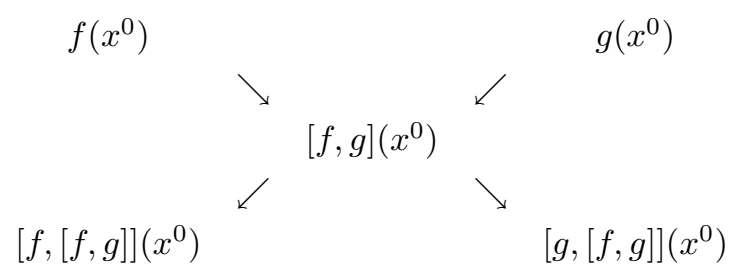

This tree includes all brackets of $f$ and $g$ up to degree three. Higher degree Lie jets are obtained similarly by repeated bracketing by $f$ and $g$. The skew symmetry and Jacobi identities induce some relations in the Lie jet, for example,

$$
[f,[g,[f, g]]]\left(x^{0}\right)=[g,[f,[f, g]]]\left(x^{0}\right)
$$

The entries of the Lie jet at $x^{0}$ are the coordinate free Taylor series coefficients of the control system around $x^{0}$ in the following sense [3], [4]. Consider a second control system

$$
\dot{z}=h(z)+k(z) u
$$

where $z \in \mathbb{R}^{p}$. If the $d$ Lie jet of (1.1) at $x^{0}$ spans $\mathbb{R}^{n}$ and there exists a linear map $T: \mathbb{R}^{n} \rightarrow \mathbb{R}^{p}$ that maps it to the $d$ Lie jet of (2.2) at $z^{0}$ term by term then there is a nonlinear map $\phi$ from a neighborhood of $x^{0}$ to a neighborhood of $z^{0}$ that almost carries trajectories to trajectories in the following sense. There exists a constant $M>0$ such that if $x(t)$ and $z(t)$ are the solutions of (1.1) and (2.2) corresponding to an admissible control $u(t)$ then

$$
|z(t)-\phi(x(t))|<M t^{d+1}
$$

Furthermore if $T$ is a linear isomorphism then $\phi$ is a local diffeomorphism. And if the systems are real analytic and $T$ preserves the infinite jets then $\phi$ is real analytic and

$$
z(t)=\phi(x(t))
$$

An analytic system is free nilpotent if its Lie jet at some state $x^{0}$ has only a finite number of nonzero brackets, these are as linearly independent as skew symmetry and 
the Jacobi identity allow and they span the tangent space at $x^{0}$. Because a free nilpotent system is nilpotent, the endpoint of a state trajectories can be found by quadratures and if the control is piecewise constant then the end point is polynomial in the switching times.

It is not hard to see that if these conditions hold at some $x^{0}$ then they hold at every $x^{1} \in \mathbb{R}^{n}$. Moreover using the above result it is not hard to see that there is an analytic diffeomorphism that carries the accessible set $\mathrm{A}\left(x^{0}\right)$ onto the accessible set $\mathrm{A}\left(x^{1}\right)$ and the fixed time accessible set $\mathrm{A}_{t}\left(x^{0}\right)$ onto the fixed time accessible set $\mathrm{A}_{t}\left(x^{1}\right)$ for any $t>0$.

If we make changes of time scale and control scale of a free nilpotent system (1.1) to obtain another system (2.2) where

$$
\begin{aligned}
& h(z)=\alpha f(z) \\
& k(z)=\beta g(z)
\end{aligned}
$$

where $\alpha>0, \beta>0$ we obtain another free nilpotent system with linearly isomorphic Lie jet. Therefore the accessible sets of (1.1) are diffeomorphic to the accessible sets of (2.2). In particular the accessible sets of (1.1) at two different times $t_{1}>0, t_{2}>0$ are diffeomorphic.

3. Linear Free Nilpotent Systems. A linear free nilpotent system (LFN) is a free nilpotent system where all brackets with two or more $g$ factors are zero. It was shown in [3] that such a system is diffeomorphic to a linear system in the usual sense. The string of integrators (1.2) is an example of a linear free nilpotent system if we add time as an explicit coordinate, $x_{0}=t$ and $\dot{x}_{0}=1$. Then at any $x^{0}$ the vectors $f\left(x^{0}\right), g\left(x^{0}\right), \operatorname{ad}_{f} g\left(x^{0}\right), \ldots, \operatorname{ad}_{f}^{n-1} g\left(x^{0}\right)$ are linearly independent and all other brackets are zero where

$$
\begin{aligned}
\operatorname{ad}_{f} g(x) & =[f, g](x) \\
\operatorname{ad}_{f}^{k} g(x) & =\left[f, \operatorname{ad}_{f}^{k-1} g\right](x)
\end{aligned}
$$

In fact

$$
\left[\begin{array}{lllll}
f\left(x^{0}\right) & g\left(x^{0}\right) & \operatorname{ad}_{f} g\left(x^{0}\right) & \ldots & \operatorname{ad}_{f}^{n-1} g\left(x^{0}\right)
\end{array}\right]=\left[\begin{array}{ccccc}
1 & 0 & 0 & & 0 \\
0 & 1 & 0 & & 0 \\
x_{1}^{0} & 0 & 1 & & 0 \\
& & & \ddots & \\
x_{n-1}^{0} & 0 & 0 & & 1
\end{array}\right]
$$

We shall continue to let $x=\left(x_{1}, \ldots, x_{n}\right)^{\prime}$.

By the result quoted in the last section the accessible sets $\mathrm{A}_{t}\left(x^{0}\right)$ and $\mathrm{A}_{t}\left(x^{1}\right)$ are diffeomorphic for any $x^{0}, x^{1}$ because the corresponding Lie jets are linearly isomorphic. 
Moreover the accessible sets $\mathrm{A}_{t}\left(x^{0}\right)$ and $\mathrm{A}_{\tau}\left(x^{0}\right)$ at two different times $t, \tau>0$ are diffeomorphic because for free nilpotent systems the Lie jets of

$$
\dot{x}=f(x)+g(x) u
$$

and

$$
\dot{x}=c(f(x)+g(x) u)
$$

are linearly isomorphic if $c \neq 0$.

Suppose we modify the linear free nilpotent system (1.2) by restricting $u \in[a, b]$ instead of $|u| \leq 1$ where $a<b$. Put another way we are considering the system

$$
\dot{x}=h(x)+k(x) v
$$

where

$$
\begin{aligned}
h(x) & =f(x)+\frac{(a+b)}{2} g(x) \\
k(x) & =\frac{(b-a)}{2} g(x) \\
|v| & \leq 1
\end{aligned}
$$

This is another linear free nilpotent system whose Lie jet at any $x^{0}$ is linearly isomorphic to that of (1.2) at any $x^{1}$ so their accessible sets are diffeomorphic.

Finally consider any linear system of the form

$$
\dot{x}=F x+G u
$$

where $x \in \mathbb{R}^{n}$ and $u \in \mathbb{R},|u| \leq 1$. For this system there are at most $n$ linearly independent brackets because

$$
\operatorname{ad}_{F x}^{k} G=F^{k} G
$$

so by Cayley Hamilton $\operatorname{ad}_{F x}^{n} G$ is linearly dependent on $G, \ldots, \operatorname{ad}_{F x}^{n-1} G$. There is a linear homomorphism from $\mathbb{R}^{n}$ to $\mathbb{R}^{n}$ that carries the Lie jet of the string of integrators (1.2) at $x^{0}$ onto the Lie jet of (3.1) at $x^{0}$ through brackets of degree $n$ so there is a smooth map from neighborhood of $x^{0}$ into itself that carries the accessible sets of (1.2) onto the accessible sets of (3.1) with an error of order $t^{n+1}$ for small $t>0$.

4. Pontryagin Maximum Principle. The main tool to compute the boundary of an accessible set is the Pontryagin Maximum Principle. If $x(s)$ is in the interior of $\mathrm{A}_{s}\left(x^{0}\right)$ at some time $s>0$ then it is in the interior of $\mathrm{A}_{t}\left(x^{0}\right)$ at all subsequent times $t>s$. Therefore if $x(t)$ is on the boundary of $\mathrm{A}_{t}\left(x^{0}\right)$ it must have been on the boundary of $\mathrm{A}_{s}\left(x^{0}\right)$ at all earlier times $0 \leq s \leq t$. Therefore if $x(t)$ is on boundary of $\mathrm{A}\left(x^{0}\right)$ the trajectory $x(0: t)$ must satisfy the Pontryagin Maximum Principle. That 
is there must exist a nontrivial adjoint trajectory $\lambda(t) \in \mathbb{R}^{n}$ such that if we define the Hamiltonian

$$
H(\lambda, x, u)=\lambda^{\prime}(f(x)+g(x) u)
$$

then

$$
\begin{aligned}
& \dot{x}(t)=\left(\frac{\partial H}{\partial \lambda}(\lambda(t), x(t), u(t))\right)^{\prime} \\
& \dot{\lambda}(t)=-\left(\frac{\partial H}{\partial x}(\lambda(t), x(t), u(t))\right)^{\prime} \\
& u(t)=\operatorname{argmax}_{|v| \leq 1} H(\lambda(t), x(t), v)
\end{aligned}
$$

A control and state trajectory that admits such a nontrivial adjoint trajectory is called an extremal.

For the above linear free nilpotent system (1.2) the adjoint dynamics (4.3) is

$$
\begin{aligned}
\dot{\lambda}_{1} & =-\lambda_{2} \\
\dot{\lambda}_{2} & =-\lambda_{3} \\
& \vdots \\
\dot{\lambda}_{n-1} & =-\lambda_{n} \\
\dot{\lambda}_{n} & =0
\end{aligned}
$$

The maximum condition (4.4) reduces to

$$
u(t)=\operatorname{sign}\left(\lambda_{1}(t)\right)
$$

provide $\lambda_{1}(t) \neq 0$. The function $\lambda_{1}(t)$ is called the switching function because at its zeros the control switches from 1 to -1 or vice versa. Controls that satisfy $|u(t)|=1$ are called bang-bang.

From the adjoint dynamics we see that the $n^{\text {th }}$ derivative of $\lambda_{1}(t)$ is zero so it is a polynomial of degree $n-1$ in $t$ and has at most $n-1$ real zeros if it is not identically zero. But the Pontryagin Maximum Principle rules out $\lambda_{1}(t)$ being identically zero because then $\lambda(t)$ is identically zero . Therefore the trajectories that lie on the boundary of the accessible set $\mathrm{A}_{t}\left(x^{0}\right)$ are generated by bang-bang controls with at most $n-1$ switches. As we discussed above these trajectories generate the boundary of $A_{t}\left(x^{0}\right)$. See [7] for more details.

5. Quadratic Free Nilpotent Systems. A system is quadratic free nilpotent (QFN) if it is free nilpotent and all brackets with three or more $g$ factors are zero. The systems (1.3) are examples when $n \geq 2 k$. When $n<2 k$ there are relations among the nonzero brackets so these systems are not free nilpotent. The index $k$ is called the order of singularity of the system. 
It follows from the Pontryagin Maximum Principle that for these systems if a control trajectory $u(t)$ generates a state trajectory $x(t)$ on the boundary of the set of accessible points of (1.3) then there exists an adjoint trajectory $\lambda(t)$ such that

$$
\begin{aligned}
\dot{\lambda}_{1} & =-\lambda_{2} \\
\dot{\lambda}_{2} & =-\lambda_{3} \\
& \vdots \\
\dot{\lambda}_{k} & =-\lambda_{k+1}-\lambda_{n+1} x_{k} \\
\dot{\lambda}_{k+1} & =-\lambda_{k+2} \\
& \vdots \\
\dot{\lambda}_{n-1} & =-\lambda_{n} \\
\dot{\lambda}_{n} & =0 \\
\dot{\lambda}_{n+1} & =0
\end{aligned}
$$

Again $\lambda_{1}$ is called the switching function because the Maximum Principle implies that if $\lambda_{1}(t) \neq 0$ then

$$
u=\operatorname{sign}\left(\lambda_{1}\right)
$$

where

$$
\operatorname{sign}\left(\lambda_{1}\right)=\left\{\begin{array}{ccc}
1 & \text { if } & \lambda_{1}>0 \\
0 & \text { if } & \lambda_{1}=0 \\
-1 & \text { if } & \lambda_{1}<0
\end{array}\right.
$$

If $\lambda_{1}(t) \neq 0$ on some open time interval $\left(t_{1}, t_{2}\right)$ then the control is bang-bang $|u(t)|=1$ but if $\lambda_{1}(t)=0$ on some closed time interval $\left[t_{1}, t_{2}\right]$ then the control is $u(t)=0$ and is said to be singular. If $\lambda_{1}(t)$ swithches sign at some $t_{1}$ then the control switches from +1 to -1 or vice versa. To determine the singular control we must repeatedly differentiate $\lambda_{1}(t)$ until $u$ appears. For the quadratic free nilpotent system (1.3) the control first appears after $2 k$ differentiations.

We are particularly interested in the case when $n=2 k$ for then the $n$ derivative of $\lambda_{1}(t)$ is

$$
\lambda_{1}^{(n)}=(-1)^{k} \lambda_{n+1} u=0
$$

so if the constant $\lambda_{n+1} \neq 0$ then the singular control is $u=0$ and the switching differential equation is

$$
\lambda_{1}^{(n)}=(-1)^{k} \lambda_{n+1} \operatorname{sign}\left(\lambda_{1}\right)
$$

It should be noted that solutions to the switching differential equation (5.3) are $C^{n-1}$ and polynomials of degree $n$ between zeros. Certainly $\lambda_{1}(t)=0$ is a solution. Moreover the switching differential equation is not locally Lipschitz continuous at 
$\lambda_{1}=0$ so solutions are not necessarily unique at this point. In fact as we shall see in a moment it is possible for the $\lambda_{1}(t)=0, t \in\left[t_{1}, t_{2}\right]$ solution to branch from or into nonzero solutions.

The switching differential equation motivates us to consider differential equations of the form

$$
\phi^{(n)}=\beta \operatorname{sign}(\phi)
$$

where $n$ may be odd or even and $\beta \neq 0$. If $\phi(t)$ is a solution of this equation then $\psi(t)=\frac{1}{|\beta|} \phi(t)$ satisfies

$$
\psi^{(n)}=\operatorname{sign}(\beta) \operatorname{sign}(\phi)
$$

so without loss of generality we can assume that $|\beta|=1$.

There are two one parameter groups that act on the set of solutions of (5.4). The first is translation in time $\tau \in \mathbb{R}$, if $\phi(t)$ is a solution then so is

$$
\psi(t)=\phi(t+\tau)
$$

The second group action is rescaling which is defined for $\alpha>0$,

$$
\psi(t)=\alpha^{n} \psi(t / \alpha)
$$

The nonlinear eigenvalues are those $\alpha>0$ such that there exists a solution $\phi(t)$ of (5.4) satisfying $\phi(0)=0$, its next zero occurs at $t=1$ and

$$
\phi(t+1)=-\alpha^{n} \phi(t / \alpha)
$$

Now $\phi(t)$ is $C^{n-1}$ and a polynomial of degree $n$ between zeros $\phi(t)$ so

$$
\phi(t)=\sum_{i=1}^{n} \frac{c_{i}}{i !} t^{i}
$$

where $c_{n}= \pm \operatorname{sign}\left(c_{1}\right)$. From the relation (5.7) and its first $n-1$ time derivatives at $t=1$ we obtain the equations

$$
\left[\begin{array}{cccccc}
1 & 1 / 2 ! & 1 / 3 ! & \ldots & 1 /(n-1) ! & 1 / n ! \\
1+\alpha^{n-1} & 1 & 1 / 2 ! & \ldots & 1 /(n-2) ! & 1 /(n-1) ! \\
0 & 1+\alpha^{n-2} & 1 & \ldots & 1 /(n-3) ! & 1 /(n-2) ! \\
& & & \ddots & & \\
& & & & & \\
& & & & & \\
0 & 0 & 0 & \ldots & 1+\alpha & 1
\end{array}\right]\left[\begin{array}{c}
c_{1} \\
\\
\vdots \\
\\
c_{n}
\end{array}\right]=\left[\begin{array}{c}
0 \\
\\
\vdots \\
\\
\\
\\
\end{array}\right]
$$


Since $c_{n}= \pm 1$ we see that $\left(c_{1}, \ldots, c_{n}\right)^{\prime}$ is a nontrivial solution to a set of homogeneous linear equations. Let $p_{n}(\alpha)$ be the determinant of the matrix in (5.9) then the nonlinear eigenvalues are the positive real roots of $p_{n}(\alpha)=0$. This polynomial is of degree $n(n-1) / 2$ but we conjecture that it has exactly $n-1$ positive real roots. We also conjecture that the positive real roots of $p_{n-1}$ interlace the positive real roots of $p_{n}$ [7]. By the way it is not hard to see if $\alpha$ is a root then so is $1 / \alpha$ and $\alpha=1$ is a root if $n$ is even, $\alpha=-1$ is a root if $n$ is odd.

Here are the first three polynomials, higher degree polynomials are presented in [7],

$$
\begin{aligned}
& p_{2}(\alpha)=(-\alpha+1) / 2 \\
& p_{3}(\alpha)=\left(\alpha^{3}-2 \alpha^{2}-2 \alpha+1\right) / 6 \\
& p_{4}(\alpha)=\left(-\alpha^{6}+3 \alpha^{5}+5 \alpha^{4}-5 \alpha^{2}-3 \alpha+1\right) / 24
\end{aligned}
$$

and here are the positive real roots of $p_{2}$ through $p_{4}$.

\begin{tabular}{l|lll}
2 & 1.0000 & & \\
3 & 2.6180 & 0.3820 & \\
4 & 4.1302 & 1.0000 & 0.2421
\end{tabular}

Corresponding to each nonlinear eigenvalue $\alpha$ is a nonlinear eigen polynomial, i.e., a polynomial (5.8) whose coefficients satisfy $c_{1}>0, c_{n}= \pm 1$ and the linear equations (5.9). Each such polynomial generates a two parameter family of solutions to (5.4) under the two group actions of time shift (5.5) and rescaling (5.6).

If $n$ is even then the quantity

$$
|\phi| \mp \phi^{(1)} \phi^{(n-1)} \pm \phi^{(2)} \phi^{(n-2)} \mp \ldots \pm(-1)^{n / 2}\left(\phi^{(n / 2)}\right)^{2} / 2
$$

is an integral of the motion

$$
\phi^{(n)}= \pm \operatorname{sign}(\phi)
$$

and if $n$ is odd

$$
\begin{array}{r}
\frac{d}{d t}\left(|\phi| \mp \phi^{(1)} \phi^{(n-1)} \pm \phi^{(2)} \phi^{(n-2)} \mp \ldots \pm(-1)^{(n-1) / 2} \phi^{((n-1) / 2)} \phi^{((n+1) / 2)}\right) \\
=(-1)^{(n-1) / 2}\left(\phi^{((n+1) / 2)}\right)^{2}
\end{array}
$$

6. QFN System Singular of Order One. The simplest quadratic free nilpotent system is the one that is singular of order one (1.3) with $n=2$ and $k=1$. The accessible sets of this system and related ones were described in [6]. We review part of that work.

Because the system is free nilpotent the accessible sets from two different initial states $\mathrm{A}_{t}\left(x^{0}\right)$ and $\mathrm{A}_{t}\left(x^{1}\right)$ are diffeomorphic. Furthermore the accessible sets at two 
different times $\mathrm{A}_{t}\left(x^{0}\right)$ and $\mathrm{A}_{\tau}\left(x^{0}\right)$ are diffeomorphic. Hence it suffices to describe the accessible set when $x^{0}=0$ and $t=1$.

We already know the projection of three dimensional accessible set $A_{1}(0)$ on to the first two coordinates as it is the accessible set of the linear free nilpotent system (1.2) of dimension $n=2$ that was described in the introduction. Its interior is the diffeomorphic image of $\mathrm{S}_{1}^{2}$ under either $\mathrm{R}_{ \pm}\left(s_{0}, s_{1}, s_{2}\right)$. Its boundary consists of the image of the interior of $\mathrm{S}_{1}^{1}$ under $\mathrm{R}_{+}\left(s_{0}, s_{1}\right)$, the image of the interior of $\mathrm{S}_{1}^{1}$ under $\mathrm{R}_{-}\left(s_{0}, s_{1}\right)$ and the two points $\mathrm{R}_{ \pm}(1)$.

Therefore the boundary of the projection is part of the boundary of three dimensional accessible set $\mathrm{A}_{1}(0)$, it is part of the zero and one dimensional boundary. For each point $\left(x_{1}, x_{2}\right)^{\prime}$ in the interior of the projection of the three dimensional accessible set $\mathrm{A}_{1}(0)$ there is a maximum and minimum value of $x_{3}$ such that $\left(x_{1}, x_{2}, x_{3}\right)^{\prime} \in \mathrm{A}_{1}(0)$. It follows from the Pontryagin Maximum Principle that the extremal trajectory that achieves the minimum $x_{3}$ for given $\left(x_{1}, x_{2}\right)^{\prime}$ must have an adjoint trajectory with $\lambda_{3}<0$ and the one that maximizes $x_{3}$ for given $\left(x_{1}, x_{2}\right)^{\prime}$ must have an adjoint trajectory with $\lambda_{3}>0$. Without loss of generality we can assume that $\lambda_{3}=-1$ for extremals minimizing $x_{3}$ and $\lambda_{3}=1$ for extremals maximizing $x_{3}$.

Therefore the switching functions for the $x_{3}$ minimizing extremals satisfy the ODE

$$
\ddot{\lambda}_{1}=\operatorname{sign}\left(\lambda_{1}\right)
$$

The quantity $\left|\lambda_{1}\right|-\dot{\lambda}_{1}^{2} / 2$ is an integral of the motion and the phase portrait is shown on the left side of Figure 2. Notice that each trajectory has exactly one switch and the total length must be $t=1$. So how can we generate a two parameter family of extremals to form the lower two dimensional bounding surface? The answer is that the phase portrait is incomplete for there are extremal trajectories consisting of a bang bang leg followed by a singular leg followed by another bang bang leg. These correspond to the two parabolas that go through the origin on the left side of Figure 2 .

On the outer bang bang legs the control could be \pm 1 while on the middle leg the control is singular, $u=0$. This generates four mappings from the interior of $\mathrm{S}_{1}^{2}$ into the two dimensional boundary of the three dimensional accessible set $\mathrm{A}_{1}(0)$. The first $\mathrm{R}_{+0+}\left(s_{0}, s_{1}, s_{2}\right)$ is the endpoint of the trajectory consisting of a bang leg of length $s_{0}$ with $u=1$, followed by a singular leg of length $s_{1}$ with $u=0$ followed by a bang leg of length $s_{2}$ with $u=1$ where $s_{0}+s_{1}+s_{2}=1$, The other three maps $\mathrm{R}_{+0-}\left(s_{0}, s_{1}, s_{2}\right)$, $\mathrm{R}_{-0+}\left(s_{0}, s_{1}, s_{2}\right)$ and $\mathrm{R}_{-0-}\left(s_{0}, s_{1}, s_{2}\right)$ are similarly defined. In $[6]$ it is shown that these four maps are diffeomorphisms on the interior of $S_{1}^{2}$ and their images do not intersect. The four images generate the two dimensional part of the lower boundary which is shown from two perspectives in Figure 3. The boundaries of these cells intersect each other at the endpoints of trajectories with one bang bang leg and one singular leg. 

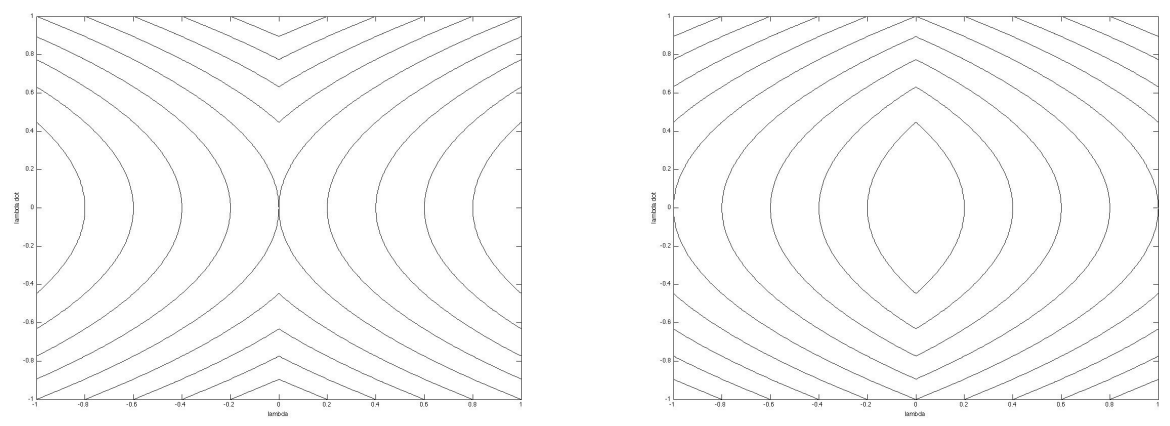

FIG. 2. Phase portraits, $\ddot{\lambda}= \pm \lambda$
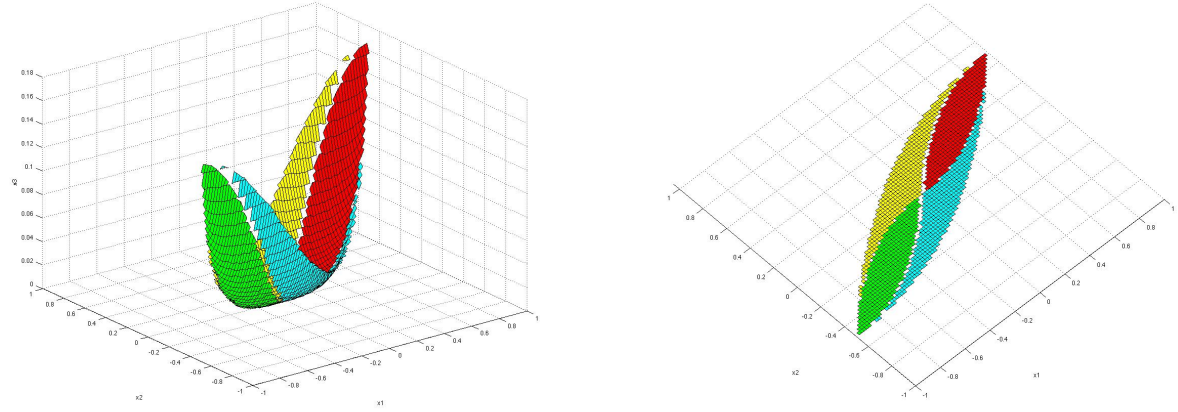

Fig. 3. Lower boundary of $\mathrm{A}_{1}(0)$

The switching functions for the $x_{3}$ maximizing extremals satisfy

$$
\ddot{\lambda}_{1}=-\operatorname{sign}\left(\lambda_{1}\right)
$$

The quantity $\left|\lambda_{1}\right|+\dot{\lambda}_{1}^{2}$ is an integral of the motion and the phase portrait is shown on the right side of Figure 2. Notice that these trajectories can have an arbitrary number of switches. There is an extremal for any sequence of switches $\left(s_{0}, \ldots, s_{k}\right)$ provided that all the interior legs are the same length, $s_{1}=s_{2}=\cdots=s_{k-1}$, and are not shorter than the outside legs, $s_{1} \geq s_{0}, s_{1} \geq s_{k}$.

But not all these extremals maximize $x_{3}$ for a given $\left(x_{1}, x_{2}\right)^{\prime}$ in the accessible set of the two dimensional linear free nilpotent system. We shall use the High Order Maximum Principle [5] to show that any trajectory with four or more bang bang legs is not extremal. Consider a trajectory with four bang bang legs of positive lengths $s_{0}, s_{1}, s_{2}, s_{3}$. From the switching equation we know this trajectory is not extremal unless $s_{0} \leq s_{1}=s_{2} \geq s_{3}$. Without loss of generality we can assume that on the first leg $u=1$. Using symbolic software it is easy to compute the endpoint of this 
trajectory,

$$
\begin{aligned}
x_{1}= & s_{0}-s_{1}+s_{2}-s_{3} \\
x_{2}= & s_{0} s_{1}+s_{2}\left(s_{0}-s_{1}\right)+s_{0}^{2} / 2-s_{1}^{2} / 2+s_{2}^{2} / 2-s_{3}^{2} / 2+s_{3}\left(s_{0}-s_{1}+s_{2}\right) \\
x_{3}= & \left(s_{3}\left(s_{0}-s_{1}+s_{2}\right)^{2}\right) / 2-\left(s_{3}^{2}\left(s_{0}-s_{1}+s_{2}\right)\right) / 2-\left(s_{0} s_{1}^{2}\right) / 2+\left(s_{0}^{2} s_{1}\right) / 2 \\
& +s_{0}^{3} / 6+s_{1}^{3} / 6+s_{2}^{3} / 6+s_{3}^{3} / 6+\left(s_{2}\left(s_{0}-s_{1}\right)^{2}\right) / 2+\left(s_{2}^{2}\left(s_{0}-s_{1}\right)\right) / 2
\end{aligned}
$$

We consider three control variations parameterized by $r_{1}, r_{2}, r_{3}$. They modify the lengths of the legs as follows

$$
\begin{aligned}
& s_{0} \leftarrow s_{0}+r_{1} / 2 \\
& s_{1} \leftarrow s_{1}-r_{1} / 2-r_{2} / 2 \\
& s_{2} \leftarrow s_{2}+r_{2} / 2+r_{3} / 2 \\
& s_{3} \leftarrow s_{3}-r_{3} / 2
\end{aligned}
$$

Notice that the $r_{i}$ can be either small positive or small negative numbers.

When $s_{2}=s_{1}$, the Jacobian of $x$ with respect to $r_{1}, r_{2}, r_{3}$

$$
\left[\begin{array}{ccc}
1 & 1 & 1 \\
2 s_{1}+s_{3} & s_{1}+s_{3} & s_{3} \\
2 s_{0} s_{1}-s_{1}^{2}-s_{3}^{2} / 2+s_{0} s_{3} & s_{0} s_{1}-s_{1}^{2} / 2-s_{3}^{2} / 2+s_{0} s_{3} & \left(s_{3}\left(2 s_{0}-s_{3}\right)\right) / 2
\end{array}\right]
$$

and its determinant is zero. The rank of this matrix is two and its null space is spanned by the vector

$$
v=\left[\begin{array}{c}
1 \\
-2 \\
1
\end{array}\right]
$$

Therefore if we combine the three variations into a single one by letting

$$
\begin{aligned}
& r_{1}=r \\
& r_{2}=-2 r \\
& r_{3}=r
\end{aligned}
$$

and denote by $x(r)$ the locus endpoints of the resulting trajectories then

$$
\begin{aligned}
& \frac{d x}{d r}(0)=0 \\
& \frac{d^{2} x}{d r^{r}}(0)=\left[\begin{array}{c}
0 \\
1 \\
s_{0}
\end{array}\right]
\end{aligned}
$$


The High Order Maximum Principle requires that if the bang bang trajectory with legs $s_{0} \leq s_{1}=s_{2} \geq s_{3}$ is extremal then the adjoint vector $\lambda(t)$ at time $t=s_{0}+s_{1}+s_{2}+s_{3}$ must satisfy

$$
\lambda^{\prime}(t) \frac{d^{2} x}{d r^{2}}(0) \leq 0
$$

The adjoint vector must be orthogonal to the columns of the matrix (6.3) and point upwards in the $x_{3}$ direction so it is

$$
w=\left[\begin{array}{c}
s_{3}^{2} / 2-s_{1} s_{3} / 2 \\
s_{1} / 2-s_{0} \\
1
\end{array}\right]
$$

Now

$$
w^{\prime} \frac{d^{2} x}{d r^{r}}(0)=s_{1} / 2>0
$$

therefore the High Order Maximum Principle is violated and the bang bang trajectory with legs $0<s_{0} \leq s_{1}=s_{2} \geq s_{3}>0$ is not an extremal.

So we have shown that to be an extremal the trajectory can have at most three legs of positive length and $0<s_{0} \leq s_{1} \geq s_{2}>0$. But not all of these extremal trajectories lie on the boundary of the set of accessible points $\mathrm{A}_{t}\left(x^{0}\right)$ because there are focal points. The two surfaces that are the images of the maps

$$
\left(s_{0}, s_{1}, s_{2}\right) \mapsto \mathrm{R}_{ \pm}\left(s_{0}, s_{1}, s_{2}\right)
$$

for $0<s_{0} \leq s_{1} \geq s_{2}>0$ intersect along the line $s_{0}+s_{2}=s_{1}$ and the locus of endpoints of the corresponding surfaces are focal points. The $\mathrm{R}_{ \pm}$surface is higher in the $x_{3}$ direction when the control on the middle leg is $u= \pm 1$. The upper boundary is shown in Figure 4.

7. QFN System Singular of Order Two. The next quadratic free nilpotent system is singular of order two, (1.3) with $n=4$ and $k=2$ which we call the extended Fuller system. The system considered by Fuller [1] is (1.3) with $n=2$ and $k=2$ but it is not free nilpotent. Fuller showed that his system had bang bang extremals that chattered, i.e., had a countably infinite number of switches in a finite time interval. This result was significant because it showed that optimal controls need not be piecewise continuous as many had conjectured. We shall focus our attention on the extended Fuller system because it has all the nice properties of a free nilpotent system described above. In particular for the extended Fuller system there are chattering boundary trajectories from every initial state $x^{0}$. This is not true for the Fuller system.

We already know the projection the five dimensional accessible set $\mathrm{A}_{1}(0)$ on to the first four coordinates as it is the accessible set of the linear free nilpotent system 


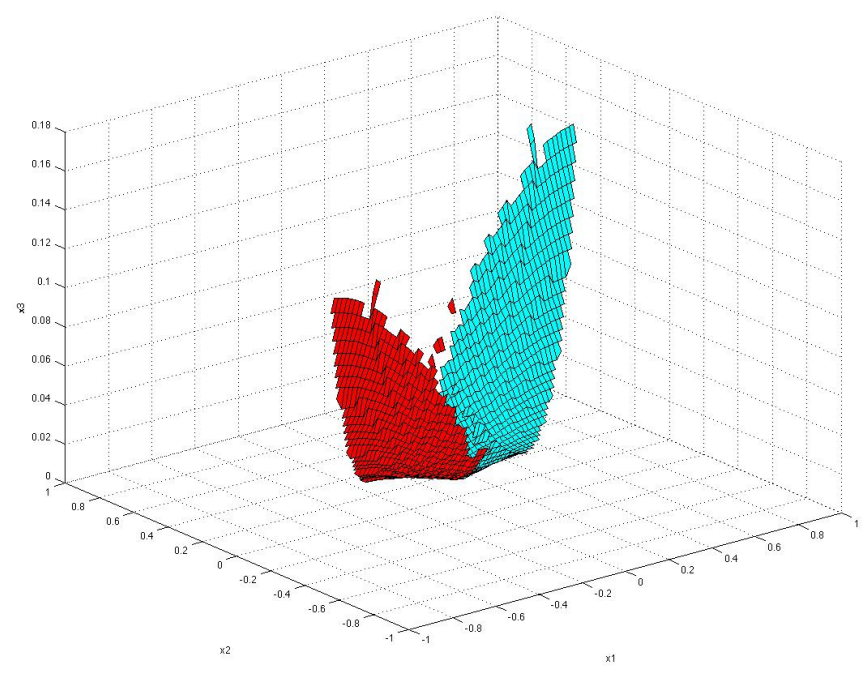

FiG. 4. Upper boundary of $\mathrm{A}_{1}(0)$

(1.2) of dimension $n=4$ that was described in the introduction. Its interior is the diffeomorphic image of $\mathrm{S}_{1}^{4}$ under either $\mathrm{R}_{ \pm}\left(s_{0}, s_{1}, s_{2}, s_{3}, s_{4}\right)$. Its boundary consists of the images of the interior of $\mathrm{S}_{1}^{3}$ under $\mathrm{R}_{ \pm}\left(s_{0}, s_{1}, s_{2}, s_{3}\right)$, the images of the interior of $\mathrm{S}_{1}^{2}$ under $\mathrm{R}_{ \pm}\left(s_{0}, s_{1}, s_{2}\right)$, the images of the interior $\mathrm{S}_{1}^{1}$ under $\mathrm{R}_{ \pm}\left(s_{0}, s_{1}\right)$, and the two points $\mathrm{R}_{ \pm}(1)$.

Therefore the boundary of the projection is part of the boundary of five dimensional accessible set $\mathrm{A}_{1}(0)$, it is part of the zero and one dimensional boundary. For each point $\left(x_{1}, x_{2}, x_{3}, x_{4}\right)^{\prime}$ in the interior of the projection of the five dimensional accessible set $\mathrm{A}_{1}(0)$ there is a maximum and minimum value of $x_{5}$ such that $\left(x_{1}, x_{2}, x_{3}, x_{4}, x_{5}\right)^{\prime} \in \mathrm{A}_{1}(0)$. It follows from the Pontryagin Maximum Principle that the extremal trajectory that achieves the minimum $x_{5}$ for given $\left(x_{1}, x_{2}, x_{3}, x_{4}\right)^{\prime}$ must have an adjoint trajectory with $\lambda_{5}<0$ and the one that maximizes $x_{5}$ for given $\left(x_{1}, x_{2}, x_{3}, x_{4}\right)^{\prime}$ must have an adjoint trajectory with $\lambda_{5}>0$. Without loss of generality we can assume that $\lambda_{5}=-1$ for extremals minimizing $x_{5}$ and $\lambda_{5}=1$ for extremals maximizing $x_{3}$.

Therefore the switching functions $\phi(t)=\lambda_{1}(t)$ for the $x_{5}$ minimizing extremals satisfy the ODE

$$
\phi^{(4)}=-\operatorname{sign}(\phi)
$$

As we discussed above the quantity

$$
|\phi|+\phi^{(1)} \phi^{(3)}-\frac{\left(\phi^{(2)}\right)}{2}
$$


is an integral of the motion. There exists two nonlinear eigenvalues $\alpha_{-} \approx 0.2421$ and its reciprocal $\alpha_{+} \approx 4.1302$. The corresponding eigen polynomials normalized to be zero at $t=0$ and $t=1$ are

$$
p_{-}(t)=0.0516 t-0.2882 t^{2} / 2+0.8051 t^{3} / 6-t^{4} / 24
$$

and its shifted time reversal

$$
p_{+}(t)=0.0516(1-t)-0.2882(1-t)^{2} / 2+0.8051(1-t) t^{3} / 6-(1-t)^{4} / 24
$$

Corresponding to each eigen polynomial there is a self similar solution to (7.1) defined by

$$
\phi_{i}(t)=\left\{\begin{array}{ccc}
p_{i}(t) & \text { if } & t \in[0,1] \\
-\alpha^{4} p_{i}\left((t-1) / \alpha_{i}\right. & \text { if } & t \in\left[1,1+\alpha_{i}\right] \\
\alpha^{8} p_{i}\left(\left(t-1-\alpha_{i}\right) / \alpha_{i}\right. & \text { if } & t \in\left[1+\alpha_{i}, 1+\alpha_{i}+\alpha_{i}^{2}\right]
\end{array}\right.
$$

and so on. Each of these solutions is acted on by the two one parameter groups of time shifting (5.5) and rescaling (5.6) to obtain a two parameter family of self similar extremals.

We can parameterize the decaying extremals corresponding to $\alpha_{-} \approx 0.2421$ by two parameters. The first is the length of time until the switching function and its first 3 time derivatives reach 0 . This will require a countably infinite number of switches. The ratio between successive intervals is $\alpha_{-}$. The second parameter is essentially a phase angle. Let $s_{0}$ and $s_{1}$ be the lengths of the first two bang bang intervals and let $u_{0}$ and $u_{1}$ be value of the control on these intervals. Then $0 \leq \alpha_{1} s_{0} \leq s_{1}$ and $u_{0} u_{1}=-1$. The the phase angle is

$$
\theta=\frac{u_{0} \alpha_{-} s_{0}}{s_{1}} \pi
$$

This angle varies between $-\pi$ and $\pi$. Therefore this family of extremals is topologically a cone with vertex where the switching functions and its first 3 time derivatives reach 0 .

Once the switching function and its first three time derivatives reach 0 then the extremal can become singular $u=0$ for an arbitrary length of time. Then the switching function can chatter away from zero along any of the two parameter family corresponding to $\alpha_{+} \approx 4.1302$. This is another cone of possibilities parameterized by the time since leaving zero and the phase angle defined by the last two legs.

Altogether there are five parameters of the resulting family of chattering to zero followed by singular followed by chattering away from zero arcs, two phase angles and three time intervals. If we constrain the sum of the three times to be 1 the end points of the corresponding trajectories sweep out a four dimensional cell that forms a part of the lower (with respect to $x_{5}$ ) boundary of $\mathrm{A}_{1}(0)$. But it is not all of the 
lower boundary as it does not reach the three dimensional part of the boundary of $\mathrm{A}_{1}(0)$ which is generated by bang bang trajectories with four legs of positive lengths $s_{0}, s_{1}, s_{2}, s_{3}$.

Notice that any nonsingular solution of the switching differential equation (7.1) must have an infinite number of zeros in both forward and backward time because the fourth derivative of $\phi$ is always of the opposite sign to $\phi$. Numerical experimentation shows that as we go forward or backward the time between switches grows. If we constantly rescale and time shift any nonsingular solution so that the next consecutive zeros are always at $t=0$ and $t=1$ then it converges to the nonlinear eigen polynomial $p_{-}(t)$ in backward time and to the eigen polynomial $p_{+}(t)$ in forward time.

Therefore we conjecture that the rest of the four dimensional lower boundary of $A_{1}(0)$ is generated by bang bang solutions corresponding to the non chattering solutions to the switching differential equation (7.1) . These can have an arbitrarily large finite number of switches as their endpoints join with the endpoints of the chattering-singular-chattering trajectories described above to form the rest of the four dimensional lower boundary of the accessible set at time one.

It is important to note that not every bang bang trajectory is minimizing $x_{5}$, some are maximizing $x_{5}$. The bang bang trajectories that maximize $x_{5}$ correspond to solutions of the switching differential equation

$$
\phi^{(4)}=\operatorname{sign}(\phi)
$$

The quantity

$$
|\phi|-\phi^{(1)} \phi^{(3)}+\frac{\left(\phi^{(2)}\right)}{2}
$$

is an integral of the motion. There is one nonlinear eigenvalue of this differential equation $\alpha_{0}=1$ with corresponding eigen polynomial

$$
p_{0}(t)=t / 24-t^{3} / 12+t^{4} / 24
$$

This generates a two parameter family of periodic solutions to the switching differential equation (7.3) under time shift (5.5) and rescaling (5.6). These solutions are a topological disk paramterized by their time between consecutive zeros and a phase angle similar to (7.2).

There are also two other interesting solutions of this differential equation

$$
\begin{aligned}
& q_{-}(t)=\left\{\begin{array}{ccc}
t^{4} / 24 & \text { if } & t \leq 0 \\
0 & \text { if } & t \geq 0
\end{array}\right. \\
& q_{+}(t)=\left\{\begin{array}{ccc}
0 & \text { if } & t \leq 0 \\
t^{4} / 24 & \text { if } & t \geq 0
\end{array}\right.
\end{aligned}
$$

Each of these generates a one parameter family of solutions under time shift (5.5) as rescaling leaves them unchanged. 
One might suspect since these solutions go to zero and come away from zero there is a possibility of inserting a singular arc of arbitrary length between them. This yields additional solutions of the switching differential equation (7.3) but one can show using the High Order Maximum Principle, in particular, the Generalized Legendre Clebsch condition that none of the resulting state trajectories lie on the boundary of the set of accessible points.

Therefore the four dimensional upper boundary is generated by bang bang trajectories generated by the solutions to the switching differential equation (7.3). The question is how many legs can these bang bang trajectories have and still remain on the boundary. We saw using the High Order Maximum Principle that for the quadratic free nilpotent singular of order one that the corresponding boundary bang bang trajectories can have at most three legs. We shall show how the High Order Maximum Principle can be used to test if the endpoint of a bang bang trajectory with more than five legs is on the boundary of the accessible set..

To do so we shall use the Lie series formula. Let $\phi_{ \pm}\left(t, x^{0}\right)$ denote the flows of the vector fields $f(x) \pm g(x)$, i.e.,

$$
\begin{aligned}
\frac{d}{d t} \phi_{ \pm}\left(t, x^{0}\right) & =f\left(\phi_{ \pm}\left(t, x^{0}\right)\right) \pm g\left(\phi_{ \pm}\left(t, x^{0}\right)\right) \\
\phi_{ \pm}\left(0, x^{0}\right) & =x^{0}
\end{aligned}
$$

If $h(x)$ is any vectorfield then we can pull $h(x)$ forward under the flow to obtain a new vector field $k(x, t)$ defined by

$$
k\left(x^{1}, t\right)=\frac{\partial \phi_{ \pm}(t, \cdot)}{\partial x}\left(x^{0}\right) h\left(x^{0}\right)
$$

where $x^{0}=\phi_{ \pm}\left(-t, x^{1}\right)$.

For analytic vector fields the Taylor series expansion of $k\left(x^{1}, t\right)$ is convergent

$$
k\left(x^{1}, t\right)=\sum_{j=0}^{\infty} a d_{f \pm g}^{i} h\left(x^{1}\right) \frac{t^{j}}{j !}
$$

This is called a Lie series.

Consider a bang bang trajectory with six legs of positive length, $s_{0}, \ldots, s_{5}$. We define a five parameter family of control variations parameterized by small positive or negative numbers $r_{1}, \ldots, r_{5}$,

$$
\begin{aligned}
& s_{0} \leftarrow s_{0}+r_{1} / 2 \\
& s_{1} \leftarrow s_{1}-r_{1} / 2-r_{2} / 2 \\
& s_{2} \leftarrow s_{2}+r_{2} / 2+r_{3} / 2 \\
& s_{3} \leftarrow s_{3}-r_{3} / 2-r_{4} / 2 \\
& s_{4} \leftarrow s_{4}+r_{4} / 2+r_{5} / 2 \\
& s_{5} \leftarrow s_{5}-r_{5} / 2
\end{aligned}
$$


Instead of computing the Jacobian of the endpoint of the state trajectory with respect to these variations it is simpler to use Lie series to compute the infinitesmal effect of these variations relative to the frame

$$
[g(x) \quad[f, g],(x) \quad[f,[f, g]](x) \quad[f,[f,[f, g]]](x) \quad[g,[f,[f,[f, g]]]](x)]
$$

where $x$ is the endpoint of the state trajectory. Because the system is free nilpotent this frame is a basis for the tangent space at $x$. The result is a $5 \times 5$ matrix whose entries depend on lengths of the legs $s_{0}, \ldots, s_{5}$. The matrix is too complicated to show here and its determinant is a polynomial of degree 10 in the lengths of the interior legs $s_{1}, \ldots, s_{4}$. The determinant is zero if any of the interior legs are of length zero so we can divide the determinant by the monomial $s_{1} s_{2} s_{3} s_{4}$ to get the six degree polynomial

$$
\begin{aligned}
& P\left(s_{1}, s_{2}, s_{3} s_{4}\right)=-\left(s_{1}^{3} s_{2}^{2} s_{3}+s_{1}^{3} s_{2}^{2} s_{4}+2 s_{1}^{3} s_{2} s_{3}^{2}+3 s_{1}^{3} s_{2} s_{3} s_{4}+s_{1}^{3} s_{2} s_{4}^{2}+s_{1}^{3} s_{3}^{3}\right. \\
& +2 s_{1}^{3} s_{3}^{2} s_{4}+s_{1}^{3} s_{3} s_{4}^{2}-3 s_{1}^{2} s_{2}^{3} s_{3}-3 s_{1}^{2} s_{2}^{3} s_{4}-8 s_{1}^{2} s_{2}^{2} s_{3}^{2}-12 s_{1}^{2} s_{2}^{2} s_{3} s_{4}-4 s_{1}^{2} s_{2}^{2} s_{4}^{2} \\
& -s_{1}^{2} s_{2} s_{3}^{3}-2 s_{1}^{2} s_{2} s_{3}^{2} s_{4}-s_{1}^{2} s_{2} s_{4}^{3}+2 s_{1}^{2} s_{3}^{4}+5 s_{1}^{2} s_{3}^{3} s_{4}+4 s_{1}^{2} s_{3}^{2} s_{4}^{2}-s_{1}^{2} s_{3} s_{4}^{3} \\
& -3 s_{1} s_{2}^{4} s_{3}-3 s_{1} s_{2}^{4} s_{4}-10 s_{1} s_{2}^{3} s_{3}^{2}-15 s_{1} s_{2}^{3} s_{3} s_{4}-5 s_{1} s_{2}^{3} s_{4}^{2}+2 s_{1} s_{2}^{2} s_{3} s_{4}^{2} \\
& -2 s_{1} s_{2}^{2} * s_{4}^{3}+6 s_{1} s_{2} s_{3}^{4}+15 s_{1} s_{2} s_{3}^{3} s_{4}+12 s_{1} s_{2} s_{3}^{2} s_{4}^{2}-3 s_{1} s_{2} s_{3} s_{4}^{3}+s_{1} s_{3}^{5} \\
& +3 s_{1} s_{3}^{4} s_{4}+3 s_{1} s_{3}^{3} s_{4}^{2}-s_{1} s_{3}^{2} s_{4}^{3}-s_{2}^{5} s_{3}-s_{2}^{5} s_{4}-4 s_{2}^{4} s_{3}^{2}-6 s_{2}^{4} s_{3} s_{4}-2 s_{2}^{4} s_{4}^{2} \\
& +s_{2}^{3} s_{3} s_{4}^{2}-s_{2}^{3} s_{4}^{3}+4 s_{2}^{2} s_{3}^{4}+10 s_{2}^{2} s_{3}^{3} s_{4}+8 s_{2}^{2} s_{3}^{2} s_{4}^{2}-2 s_{2}^{2} s_{3} s_{4}^{3}+s_{2} s_{3}^{5} \\
& \left.+3 s_{2} s_{3}^{4} s_{4}+3 s_{2} s_{3}^{3} s_{4}^{2}-s_{2} s_{3}^{2} s_{4}^{3}\right) / 288
\end{aligned}
$$

The meaning of this polynomial is as follows. If a bang bang trajectory with six or more legs of positive length is an extremal of the quadratic free nilpotent system singular of order two then the lengths of any four consecutive interior legs must be a root of $P$. In other words if we have a bang bang solution of either switching differential equation (7.1) or (7.3) then the lengths of any four consecutive interior legs must be a root of $P$.

Suppose we look for roots of $P$ where the lengths of the legs form a geometric progression, $s_{2}=\alpha s_{1}, s_{3}=\alpha s_{2}, s_{4}=\alpha s_{3}$ then it turns out that $\alpha$ must be one of the nonlinear eigenvalues $\alpha_{-}, \alpha_{0}, \alpha_{+}$that we discussed above.

To test if a bang bang trajectory with six or more legs of positive length could be on the lower or upper four dimensional boundary of the accessible set we proceed as follows. First we compute the endpoint $x\left(s_{0}, s_{1}, s_{2}, s_{3}, s_{4}, s_{5}\right)$ of a bang bang trajectory with six legs using a symbolic software package. We used MATLAB's Symbolic Toolbox. Because the system is free nilpotent this is easy to do because it only involves quadratures. The result is to messy to display here. Then make the control variations (7.5) so that the endpoint is also a function of $r_{1}, \ldots, r_{5}$. Symbolically compute the Jacobian of the five dimensional end point with respect to the five variation parameters and evaluate this Jacobian at $r_{1}=r_{2}=r_{3}=r_{4}=r_{5}=0$. 
Compute a nonzero vector $v$ in the null space of the Jacobian and another nonzero vector $w$ in the null space of its transpose. Let $r_{i}=v_{i} r$ and substitute these into the formula for the endpoint. Then the first derivative of the endpoint with respect to the parameter $r$ at $r=0$ is zero. We have constructed a high order variation and we can test the second derivative of the endpoint with respect to $r$ at $r=0$ against the adjoint variable $\lambda$ at the end of the trajectory. Since the adjoint variable must annihilate all first order variations it must be a multiple of $w$. To test if the endpoint is on the lower boundary of the accessible set we take a multiple of $w$ whose fifth coordinate is -1 and to test if it is on the upper boundary we take a multiple of $w$ whose fifth coordinate is 1 .

We have applied that method to the eigen solutions of the switching differential equations (7.1) and (7.3). The test did not rule out bang bang trajectories with six or more legs corresponding to $\alpha_{ \pm}$being on the lower boundary but it did rule out bang bang trajectories with six or more legs corresponding to $\alpha_{0}$ being on the upper boundary.

We conjecture that the lower boundary is generated by all solutions of the switching differential equation (7.1) including those that chatter into zero, stay at zero for awhile and then chatter out. These solutions can have any number of legs of positive length from one to countably infinite. To be in the interior of the lower boundary they must have five or more legs .

We conjecture that the upper boundary is generated by some solutions of the switching differential equation (7.3) and they have at most five bang bang legs of positive length. But not every bang bang trajectory with five legs is on the upper boundary as there are focal points. We conjecture there are two families of bang bang trajectories generated by solutions to (7.3) with five positive legs. One family starts with $u=1$ and the other starts with $u=-1$. When constrained to total time length one these families generate two four dimensional surfaces which intersect in a three dimensional surface of focal points.

8. Conclusion. We have shown that the accessible sets of some free nilpotent systems are cell complexes which are relatively easy to describe. We reviewed the results of [7] where the accessible sets of linear free nilpotent systems are given and the results of [6] where the accessible set of a quadratic free nilpotent system singular of order one is given. We showed how the High Order Maximum Principle could be used to test whether a bang bang trajectory could be on the boundary of the accessible set. The High Order Maximum Principle has been used to test singular extremals and this may be the first time it is used to test bang bang extremals. Finally we offered some conjectures about the accessible set of a quadratic free nilpotent system singular of order two called the extended Fuller system. 


\section{REFERENCES}

[1] A. T. FUlLER, Relay control systems optimized for various performance criteria. Automatic and Remote Control, (Proc. First World Congress IFAC, Moscow, 1960), volume 1, Butterworths, London, pages 510-519, 1961.

[2] A. J. Krener, A generalization of the Pontryagin Maximum Principle and the Bang-Bang Principle. PhD Dissertation, UC Berkeley, 1971.

[3] A. J. Krener, On the equivalence of control systems and the linearization of nonlinear systems. SIAM Journal on Control, 11(1973), pp. 670-676.

[4] A. J. KRener, Local approximation of control systems. Journal of Differential Equations, 19(1975), pp. 125-133.

[5] A. J. KRener, The high order maximal principle and its application to singular extremals. SIAM Journal on Control and Optimization, 15(1977), pp. 256-293.

[6] A. J. Krener and H. Schaettler, The structure of small time reachable sets in low dimensions, SIAM Journal on Control and Optimization, 27(1988), pp. 120-147.

[7] A. J. Krener, Accessible sets of free nilpotent control systems. To appear in the proceedings of NOLCOS 2010.

[8] H. Sussmann And V. Jurdjevic, Control of nonlinear systems. Journal of Differential Equations, 12(1972), pp. 95-116.

[9] M. I. Zelikin And V. F. Borisov, Theory of Chattering Control. Birkhauser, Boston, 1994. 